107 Royal Netherlands Institute for Sea Research

This is a postprint version of:

Villanueva, L., Sinninghe Damsté, J. S., \& Schouten, S. (2014). A reevaluation of the archaeal membrane lipid biosynthetic pathway. Nature Reviews Microbiology, 12(6), 438-448.:

Published version: http://dx.doi.org/10.1038/nrmicro3260

Link NIOZ Repository: www.vliz.be/nl/imis?module=ref\&refid=239934

[Article begins on next page]

The NIOZ Repository gives free access to the digital collection of the work of the Royal Netherlands Institute for Sea Research. This archive is managed according to the principles of the Open Access Movement, and the Open Archive Initiative. Each publication should be cited to its original source - please use the reference as presented.

When using parts of, or whole publications in your own work, permission from the author(s) or copyright holder(s) is always needed. 


\title{
A Re-evaluation of the Archaeal Membrane Lipid Biosynthetic Pathway
}

\author{
Laura Villanueva, Jaap S. Sinninghe Damsté, \\ and Stefan Schouten
}

NIOZ, Royal Netherlands Institute for Sea Research. Department of Marine Organic Biogeochemistry. P.O. Box 59, NL-1790 AB Den Burg, The Netherlands. Correspondence to L.V. e-mail: laura.villanueva@nioz.n1

Note: Terms highlighted in grey are included in the glossary. 


\section{Abstract}

2 Archaea produce unique membrane lipids in which isoprenoid alkyl chains are bound

3 through ether linkages to glycerol moieties. With the increasing availability of cultured

4 representatives of the archaea over the past decade, archaeal genomic and membrane

5 lipid composition data have become available. Here, we compare the amino acid

6 sequences of the key enzymes of the archaeal ether lipid biosynthesis pathway and

7 criticality evaluate past studies on the biochemical functioning of these enzymes.

8 Considering these evidences we propose an alternative archaeal lipid biosynthetic

9 pathway based on a multiple-key, multiple-lock mechanism.

10 In 1977 Woese \& Fox ${ }^{1}$ proposed a new domain of life, the Archaea (at that time called

11 archaeabacteria), in addition to the Eubacteria (Bacteria) and Eukarya. Although

12 initially it was thought that archaea were confined to extreme environments (e.g. high

13 temperature or salinity, extreme $\mathrm{pH}$ ), subsequent studies have shown that they occur

14 ubiquitously in non-extreme settings, such as the ocean ${ }^{2}$, where they can be substantial

15 contributors to total microbial biomass ${ }^{3}$. Archaea have now been shown to play

16 important roles in global biogeochemical cycles such as the methane ${ }^{4}$ and nitrogen ${ }^{5}$

17 cycles.

18 In addition to their genomic make-up, the domain Archaea has also other traits that

19 distinguish them from Bacteria and Eukarya. One of the most intriguing is the unique

20 structure of their membrane lipids ${ }^{6}$. Bacterial and eukaryotic membranes are composed

21 of fatty acid chains that are linked to the glycerol moiety through ester bonds. These

22 bacterial and eukaryotic lipids are organized in a bilayer structure. In contrast, archaeal

23 membrane lipids are characterized by (i) ether instead of ester linkages between the

24 glycerol moiety and the alkyl chains, (ii) isoprene-based alkyl chains instead of acetate- 
based straight alkyl chains as building blocks of the apolar side-chains, and (iii) an opposite stereochemistry of the glycerol phosphate backbone, i.e. sn-glycerol-1phosphate $(\mathrm{G} 1 \mathrm{P})^{7}$. Soon after the discovery of archaeal membrane ether lipids, it was suggested that they could provide an advantage in surviving in extreme environments (e.g. high temperature, high salinity or extreme $\mathrm{pH})^{7}$, based on the fact that the ether-

30 linked lipids present in Archaea are chemically more stable than the ester-linked lipids

31 present in Bacteria and Eukarya ${ }^{8}$. This is most likely due to restrictions in the hydrocarbon chain mobility in ether-linked membranes which also may result in reduced permeability of this membranes. However, the discovery of ether lipids in

34 ubiquitous mesophilic/neutrophilic archaea found in the ocean ${ }^{9}$ suggested that this hypothesis needed to be re-evaluated.

In addition to an exact answer as to why archaea are producing ether membrane lipids, we also lack an answer to the important question of how they biochemically produce them. Many steps in the archaeal membrane lipid biosynthetic pathway are still unknown and most studies have focused mainly on evolutionary processes involved in

40 the differentiation of bacterial and archaeal membranes ${ }^{6}$. Phylogenetic analyses of the 41 enzymes involved in the archaeal membrane lipid biosynthetic pathway have been 42 performed $^{10,11}$ but were limited to a small number of archaeal genomes available. In light of the recent availability of many more archaeal genome sequences, in particular of members of mesophilic and environmentally important archaea, and the much more detailed information available on membrane lipid composition of archaea ${ }^{12}$, it is timely to analyze the relationship between archaeal membrane ether lipid composition and the enzymes involved in their biosynthesis. The analysis of amino acid sequences of key biosynthetic enzymes presented here, as well as a critical evaluation of the current conception of the archaeal membrane ether lipids biosynthetic pathway based on 
enzymatic studies in specific archaeal isolates, indicates that the concept of the archaeal membrane lipid biosynthesis pathway has to be reconsidered.

\section{Archaeal phylogeny and membrane lipids}

Initial studies based on 16S rRNA gene sequences originally supported a deep split within the Archaea forming two major phyla: Crenarchaeota and Euryarchaeota ${ }^{13}$. Based on culture studies and the analysis of environmental gene sequences Crenarchaeota are thought to consist mostly of hyperthermophiles and thermoacidophiles $^{14}$. Most hyperthermophilic Crenarchaeota have been isolated from geothermally heated soils or waters, sulfur-rich springs, or hydrothermal vents, where they obtain their energy mainly from sulfur-containing compounds ${ }^{15}$. Euryarchaeota are abundant in a wide range of environments and have widely diverse physiological strategies (e.g. halophilic, thermophilic, methanogenic ${ }^{16}$ ). Horizontal gene transfer (HGT) is thought to have been especially important in the evolution of certain members of the Euryarchaeota. For example, Halobacteriales have acquired several genes from Bacteria and it has been proposed that HGT transformed a methanogen into the common ancestor of the Halobacteria ${ }^{17}$. The evolution of another order of the Euryarchaeota, the Thermoplasmatales, is believed to have involved extensive HGT from Sulfolobales (hyperthermophilic Crenarchaeota) and Bacteria ${ }^{18-19}$. In the last decade several other archaeal phyla have been discovered, e.g. Korarchaeota and Nanoarchaeota ${ }^{20-21}$, Thaumarchaeota ${ }^{22}$, and the recently proposed 'Aigarchaeota' phylum ${ }^{23}$. Species of the Korarchaeota, Nanoarchaeota and 'Aigarchaeota' have a limited environmental distribution, being mainly found in hot springs, and their physiology is not clear (e.g. REF 20). Thaumarchaeota, by contrast, are widespread in marine, lacustrine and terrestrial environments, as revealed by environmental genomics ${ }^{24}$. 
Although there is a wide variety of archaeal membrane lipids ${ }^{25}$, they typically feature a variation of two main core structures, i.e. sn-2,3-diphytanylglycerol diether (archaeol) with phytanyl $\left(\mathrm{C}_{20}\right)$ chains in a bilayer structure, and sn-2,3-dibiphytanyl diglycerol tetraether (also known as glycerol dibiphytanyl glycerol tetraether, GDGT), in which the two glycerol moieties are connected by two $\mathrm{C}_{40}$ isoprenoid chains, allowing the formation of monolayer membranes ${ }^{26-27}$. GDGTs can contain $0-8$ cyclopentane moieties (i.e. GDGT-x, $\mathrm{x}$ equals the number of cyclopentane moieties; REF 12; Table 1). The presence of these cyclopentane moieties is thought to be essential in maintaining functional membranes and cellular homeostasis in situations of extreme $\mathrm{pH}$ or thermal stress; the number of cyclopentane moieties increases as growth temperature increases ${ }^{28}$ and $\mathrm{pH}$ decreases ${ }^{29-30}$.

Comparison of an archaeal reference phylogeny with the membrane lipid composition distribution shows that most lipids are not specific for a certain phylogenetic group (Table 1; REF 12). Only the GDGT crenarchaeol ${ }^{9}$, containing four cyclopentane moieties and a cyclohexane moiety, is considered to be characteristic of the Thaumarchaeota ${ }^{31}$, suggesting that the biosynthesis of the cyclohexane moiety is unique for this phylum. GDGTs are the dominant lipid species in Crenarchaeota and Thaumarchaeota, while euryarchaeotal orders synthesize archaeol (Methanococcales, Halobacteriales, Methanosarcinales), GDGTs (Methanopyrales, Thermoplasmatales, Archaeoglobales, Methanomicrobiales), or both (Thermococcales, Methanobacteriales) (Table 1). GDGT-0 is found in all (hyper)thermophilic Crenarchaeota and several thermophilic euryarchaeotal orders, in some mesophilic methanogenic Euryarchaeota, and in Thaumarchaeota. GDGTs with 1-4 cyclopentane moieties are synthesized by hyperthermophilic Crenarchaeota, Thaumarchaeota, in the thermophilic euryarchaeotal 
100 (member of the DHVE-2 cluster, closely related to the Thermoplasmatales order) ${ }^{32}$.

101 However, they are apparently not synthesized by methanogenic Euryarchaeota (Table

102 1). GDGTs with more than four cyclopentane moieties (GDGTs 5-8, Table 1) are rare

103 and only found in hyperthermophilic Crenarchaeota and some hyperthermophilic

104 Euryarchaeota of the Thermoplasmatales order. GDGTs are absent in Halobacteriales

105 (Euryarchaeota) that mainly contain archaeol or extended archaeol with one $\mathrm{C}_{25}$

106 isoprenoid chain ${ }^{33}$.

\section{Archaeal lipid synthesis}

108 Previous studies have characterized some of the enzymes involved in the biosynthesis

109 of archaeal membrane ether lipids (FIG. 1). Isopentenyl diphosphate and dimethylallyl

110 diphosphate (DMAPP) serve as basic building blocks of the isoprenoid chains and are

111 synthesized by the mevalonate pathway $^{6,34}$. DMAPP is thought to be consecutively

112 condensed with several isopentenyl diphosphate units to form geranylgeranyl

113 diphosphate $\left(\mathrm{GGPP}, \mathrm{C}_{20}\right)$ by a short -chain $\left(\mathrm{C}_{20}\right)$ isoprenyl diphosphate synthase, GGPP

114 synthase (FIG. 1). The subsequent ether bond formation is catalyzed by two

115 prenyltransferases: GGPP is attached to the glycerol-1-phosphate (G1P) to form

116 geranylgeranylglyceryl phosphate (GGGP) catalyzed by the GGGP synthase. The

117 attachment of the second side chain to GGGP generates digeranylgeranylglyceryl

118 phosphate (DGGGP) and is catalyzed by the DGGGP synthase (FIG. 1). This is thought

119 to be followed, after addition of a polar headgroup to the glycerol moiety, by a

120 reduction of the unsaturated isoprenoid chains mediated by geranylgeranyl reductases ${ }^{6}$,

121 forming archaeol. The formation of GDGTs is thought to initially involve a coupling of

122 two archaeol molecules through head-to-head condensation of the phytanyl chains (FIG.

123 1). Cyclopentane moieties are subsequently thought to be formed by internal 
124 cyclization. These latter two steps are highly unusual since they involve non-activated

125 methyl groups and the enzymes involved are unknown ${ }^{6,35}$.

126 Evidence for the head-to-head coupling of archaeol comes from pulse-chase

127 experiments with cell extracts of the euryarchaeon Thermoplasma acidophilum

128 (Thermoplasmatales order) incubated with ${ }^{14} \mathrm{C}$-mevalonate, which showed incorporation

129 of radioactivity first into archaeol and then into GDGT- $0^{36}$. Furthermore, pulse-chase

130 experiments performed with cell extracts of T. acidophilum labeled with ${ }^{14} \mathrm{C}$ -

131 mevalonate and using a squalene epoxidase inhibitor (terbinafine) led to accumulation

132 of archaeol, with a modified headgroup, rather than GDGTs ${ }^{37}$. These experiments

133 suggest archaeol as the precursor of GDGTs. However, Poulter et al. ${ }^{38}$ studied the in

134 vivo incorporation of radiolabeled archaeol into cells of the euryarchaeon

135 Methanospirillum hungatei (order Methanomicrobiales) and found no incorporation of

136 radioactivity in GDGT-0. Furthermore, radiolabeled phytol, in which there is one

137 double bond, was not incorporated into archaeol and GDGT-0, while geranylgeraniol

138 was efficiently incorporated into both. Similar results were obtained by incorporation of

139 deuterium-labeled DGGGP analogs in Methanothermobacter thermoautotrophicus

140 (order Methanobacteriales) ${ }^{39-40}$. The deuterium-labeled DGGGP analogs with a terminal

141 double bond or with a saturated terminal isoprene unit were not incorporated into

142 GDGT-0, and only the DGGGP analog with a terminal isopropylidene group was

143 incorporated into the GDGT. These studies thus suggest that the presence of double

144 bonds in the DGGGP molecule is a prerequisite for the formation of GDGTs, which

145 contradicts the idea that fully saturated phytanyl chains are coupled.

146 Below we focus on three known key enzymes in the formation of glycerol ether

147 lipids formation, i.e. GGPP, GGGP, and DGGGP synthases. We searched for

148 homologues of those enzymes in all archaeal genomes available up to date, compared 
149 the amino acid moieties involved in the selection of the substrate, used maximum

150 likelihood analyses to reveal their phylogeny, and compared this with the distribution of

151 ether membrane lipids (Table 1).

\section{Isoprenyl diphosphate synthase}

153 Isoprenyl diphosphate (IPP) synthases catalyze consecutive condensations of

154 isopentenyl diphosphates with allylic primer substrates to form isoprenoid compounds,

155 including steroids, triterpenoids, carotenoids, prenylated proteins and quinones ${ }^{41}$. IPP

156 synthases synthesize short (i.e. $\left.\mathrm{C}_{10}-\mathrm{C}_{20}\right)$ or longer $\left(>\mathrm{C}_{20}\right)$ prenyl groups. IPP synthases

157 harbour two conserved aspartate-rich motifs typical of prenyltransferases, which form a

158 deep hydrophobic cleft or substrate-binding pocket ${ }^{42}$. Short-chain (up to $\mathrm{C}_{20}$ ) IPP

159 synthases are characterized by the presence of 'bulky' amino acids, i.e. phenylalanine

160 (F) or tyrosine $(\mathrm{Y})$, as the $5^{\text {th }}$ amino acid residue before the first aspartate-rich motif,

161 which limits the degree of isoprenoid chain elongation to the 20 carbon atoms of the

$162 \mathrm{GGPP}^{42}$. Some IPP synthases are flexible in the chain length they synthesize, e.g. the

163 single bifunctional short-chain IPP synthase of $M$. thermoautotrophicus synthesizes

164 both the $\mathrm{C}_{15}$ precursor for the synthesis of squalene and GGPP $\left(\mathrm{C}_{20}\right)$ for the synthesis of

165 archaeal membrane lipids ${ }^{43}$.

166 We searched for homologues of IPP synthases in 43 archaeal genomes (Table S1).

167 Some of the identified sequences harbor a small amino acid residue (alanine, A; valine,

$168 \mathrm{~V}$; serine, $\mathrm{S})$ in the $5^{\text {th }}$ amino acid residue before the first aspartate-rich motif,

169 classifying them as putative long-chain IPP synthase (Table S1). Long-chain IPP

170 synthases were only detected in species of the Thaumarchaeota phylum, in most orders

171 of the Crenarchaeota, and in the orders Halobacteriales, Methanosarcinales,

172 Archaeoglobales and Thermoplasmatales of the Euryarchaeota (Table S1). The role of

173 the long-chain IPP synthase in these groups is unknown but it has been hypothesized 
174 that is related to the synthesis of isoprenoid chains other than for ether lipids ${ }^{34,44}$, such

175 as respiratory quinones ${ }^{45}$.

176 Putative short-chain IPP synthases harboring a 'bulky' amino acid residue (Y or F)

177 at position 5 (FIG. 2) were detected in all the archaeal orders (Table S1), suggesting that

178 the archaeal lipid biosynthetic pathway starts with the formation of isoprenoid chains

179 with 20 carbon atoms (GGPP). According to the current picture of the archaeal lipid

180 biosynthetic pathway (FIG. 1), short-chain IPP synthases should always encounter the

181 same substrate (isopentenyl diphosphate units) and yield the same product, i.e. GGPP.

182 However, the substantial differences between IPP synthases at the amino acid level (e.g.

183 FIG. 2) seems at odds with this idea. Rather, the observed large amino acid sequence

184 variability of the IPP synthases and, thus the expected plasticity in the structure of this

185 enzyme suggests structural diversity in the intermediates synthesized from isopentenyl

186 diphosphate units.

187 Geranylgeranylglyceryl phosphate synthase

188 The next step in the proposed biosynthetic pathway consists of the formation of an ether

189 linkage between the C-3 of the G1P and GGPP to form GGGP (FIG. 1). This step is

190 mediated by the GGGP synthase, which is selective for the G1P acceptor but also for

191 the isoprenoid chain added, strongly favoring GGPP over shorter or longer chains ${ }^{47}$.

192 GGGP synthase represents the first identified triose phosphate isomerase (TIM) barrel

193 structure with a prenyltransferase function, which is thought to be unique to the

$194 \operatorname{archaea}^{48}$. GGGP synthase is a homologue of PcrB protein that catalyzes the

195 condensation of G1P with $\mathrm{C}_{35}$ heptaprenyl pyrophosphate (HepPP) to

196 heptaprenylglyceryl phosphate (HepGP) in Gram-positive bacteria (e.g. Bacillus

197 subtilis $)^{49}$. 
The only GGGP synthase characterized in detail so far is that of the euryarchaeon

199 Archaeoglobus fulgidus ${ }^{48}$, which produces archaeol and GDGT-0 as membrane lipids

200 (Table 1). The crystal structure of this enzyme displays a unique fold acting as a 'greasy

201 slide' and a 'swinging door' due to the replacement of a helix $\alpha-3$ by a strand that

202 creates a large gap for the product of IPP synthase to fit in ${ }^{48}$. It is thought that a 'bulky'

203 hydrophobic amino acid residue, i.e. tryptophan (W), at position 99 ( $\alpha 4$ a helix of $A$.

204 fulgidus; here referred to as the 'chain-length determination area'; FIG. 3), usually

205 marks the end of the gaps in the barrel and would presumably select for the chain length

206 of the substrate (in this case presumably GGPP, $\mathrm{C}_{20}$ ). The GGGP synthase of $A$. fulgidus

207 and PcrB from B. subtilis share $35 \%$ sequence identity and the binding sites for G1P are

208 conserved (FIG. 3; REF 49). Interestingly, the residue corresponding to alanine (A) at

209 position $100\left(\mathrm{~A}_{100}\right)$ in PcrB from Bacillus, as well as the tyrosine (Y) 104, allow the

210 binding of substrates longer than GGPP, i.e. $>\mathrm{C}_{20}$ (REF 50). This $\mathrm{A}_{100}$ residue

211 corresponds to $\mathrm{W}_{99}$ in the A. fulgidus IPGP synthase (FIG. 3). The conversion of $\mathrm{A}_{100}$ to

$212 \mathrm{~W}_{100}$ in PcrB from Bacillus has been proven to prevent the formation of $\mathrm{C}_{35}$ products,

213 and the one from $\mathrm{Y}_{104}$ to $\mathrm{A}_{104}$ to allow the formation of longer products up to $\mathrm{C}_{40}(\mathrm{REF}$

214 50). Guldan et al. ${ }^{49}$ also showed that the conversion of $\mathrm{W}_{99}$ to $\mathrm{A}_{99}$ in the A. fulgidus

215 GGGP synthase allowed the protein to use substrates longer than GGPP.

216 We searched for GGGP synthase homologues in 72 archaeal genomes and aligned

217 them with the GGGP synthase sequences from A. fulgidus (simplified alignment in FIG.

218 3). Interestingly, the 'bulky' $\mathrm{W}_{99}$ amino acid residue found in A. fulgidus GGGP

219 synthase, which is believed to restrict the length to $\mathrm{C}_{20}$ substrates, was only detected in

220 sequences of the euryarchaeotal orders Archaeoglobales, Halobacteriales and

221 Methanomicrobiales, while in the remaining sequences either a glycine $(\mathrm{G})$ or alanine

222 (A), both small amino acid residues, were found in the corresponding position. This 
amino acid position also coincides with the presence of the $\mathrm{A}_{100}$ residue found in PcrB

224 of Bacillus, which allows it to use longer $\left(>\mathrm{C}_{20}\right)$ isoprenyl chains as substrates. Indeed,

225 the secondary structure of the partial amino acid sequences (FIG. 3) showed that the

$226 \mathrm{G} / \mathrm{A}_{99}$ residue observed in most archaeal sequences (other than the euryarchaeotal

227 orders Archaeoglobales, Halobacteriales and Methanomicrobiales) was included in an

$228 \alpha$-helix structure as in the case of $\mathrm{W}_{99}$ of $A$. fulgidus ( $\alpha 4 \mathrm{a}$ helix according to ${ }^{48}$ ).

229 Moreover, the amino acid sequence alignment of GGGP synthases (FIG. 3), also reveals

230 the presence of a 'bulky' tryptophan (W) residue in the $\alpha 5$ ' helix (as defined for $A$.

231 fulgidus) in all the thaumarchaeotal sequences (data not shown), while in the

232 corresponding position in the rest of the sequences there is a small amino acid residue

233 (glycine, $\mathrm{G}$ or alanine, A). In fact, the protein secondary structure analysis does not

234 predict the existence of an $\alpha$-helix in this position in the archaeal GGGP synthases other

235 than A. fulgidus and the PcrB of Bacillus subtilis (FIG. 3). This amino acid change in

236 the thaumarchaeotal sequences would certainly affect the positioning of the isoprenyl

237 substrate in the GGGP synthase TIM-barrel structure.

238 These key differences in the amino acid composition of GGGP synthases suggest

239 that their structure, as well as the amino acid interactions between the isoprenyl

240 substrate and the TIM-barrel structure of the GGGP synthase, are likely to be quite

241 different from the enzyme characterized in the euryarchaeon A. fulgidus. Our analysis of

242 the amino acid sequence diversity of archaeal GGGP synthases strongly suggests that

243 they harbor functional plasticity and enable the selection of substrates that are longer

244 than GGPP.

245 The phylogeny of GGGP synthase reveals two main clusters (FIG. 4). Cluster 1

246 includes the euryarchaeotal orders Archaeoglobales, Methanomicrobiales and

247 Halobacteriales while cluster 2 can be further subdivided into cluster $2 \mathrm{~A}$, which 
248 includes the Thaumarchaeota and the Crenarchaeota, and cluster 2B, which includes the

249 remaining euryarchaeotal groups. The large difference between the three euryarchaeotal

250 orders in cluster 1 and the other Archaea (FIG. 4) has been previously related to the

251 presence of an ancestral divergent type of GGGP synthase in Halobacteria ${ }^{10}$.

252 Interestingly, GGGP synthase sequences seem to roughly cluster according to the

253 presence/absence of ring moieties in the membrane lipids with the notable exception of

254 GGGP synthases from the Thermoplasmatales order. However, the phylogenetic positioning of GGGP synthases of the Thermoplasmatales order has probably been strongly affected by events of vertical inheritance from an euryarchaeotal ancestor ${ }^{18}$.

\section{Digeranylgeranylglyceryl phosphate synthase}

258 The next step in the proposed pathway consists of the catalysis of GGGP by the

259 DGGGP synthase to form DGGGP (FIG. 1). DGGGP synthase is a member of the UbiA

260 prenyltransferase family, which, apart from being involved in the archaeal ether lipid

261 formation, also transfers prenyl groups to hydrophobic ring structures such as quinones, 262 hemes, chlorophylls, vitamin E, or shikonin ${ }^{56}$.

263 We searched for putative DGGGP synthases in archaeal genomes based on protein

264 homology with the DGGGP synthase of the crenarchaeota Sulfolobus solfataricus

265 which function has been previously tested experimentally ${ }^{56}$. DGGGP synthases were

266 highly divergent between archaeal orders and no clustering was observed (FIG. 5). The

267 most striking observation, however, is the lack of homologues of DGGGP synthases in

268 the Thaumarchaeota, as observed previously for a more limited database ${ }^{34}$. However,

269 several putative protoheme IX farnesyltransferases and other prenyltransferases were

270 identified in thaumarchaeotal genomes (FIG. 5). The inability to clearly identify

271 DGGGP synthases in thaumarchaeotal genomes suggests the existence of very

272 divergent DGGGP synthases in this phylum compared to others. Interestingly, 
273 Thaumarchaeota are the only archaea capable of biosynthesizing GDGTs containing a

274 cyclohexane moiety (crenarchaeol). Sinninghe Damsté et al. ${ }^{9}$ showed that this

275 additional cyclohexane ring led to a 'bulge' in one of the biphytanyl chains that

276 prevents the dense packing of the biphytanyl chains in the thaumarchaeotal GDGT

277 membranes. Possibly, this 'bulky' biphytanyl chain can only be accommodated by a

278 DGGGP synthase that is rather different from those using regular biphytanyl chains as

279 substrates.

280 An alternative pathway for ether lipid biosynthesis

281 Our results, together with the, sometimes contradicting, circumstantial evidences on e.g.

282 the substrates utilized for formation of GDGTs (REF 37 vs REFs 38, 40), are difficult to

283 reconcile with the current ether membrane lipid biosynthetic pathway (FIG. 1). We,

284 therefore, propose an alternative pathway that better explains our, and earlier ${ }^{6}$,

285 observations, while at the same time circumventing some unresolved issues (i.e. head-

286 to-head condensation of saturated phytanyl chains; ring formation) in the currently

287 proposed pathway. The new hypothetical pathway is based on a multiple-key, multiple-

288 lock mechanism for which multiple keys with different structures, due to the

289 presence/absence of rings, must accommodate and specifically interact at the molecular

290 level with different locks (i.e. GGGP and DGGGP synthases) (FIG. 6). The large

291 difference in amino acid sequences of IPP, GGGP and DGGGP synthases indicate a

292 larger functional plasticity than previously anticipated. One explanation could be that

293 the rings are already present in the prenyl chains before they are coupled to the glycerol

294 unit (for GGGP and DGGGP synthases). Formation of ring structures at this early stage

295 would avoid the need to form them by internal cyclization of saturated chains. 
296 Potentially this cyclization may happen simultaneously with the chain elongation using 297 isopentenyl diphosphate (FIG. 6).

298 The presence of small amino acid residues in the chain-length determination area of 299 archaeal GGGP synthases (cluster 2; FIG. 4) indicates that these synthases could 300 accommodate prenyl substrates longer than $\mathrm{C}_{20}$. This implies that the substrates of

301 GGGP synthases could be $\mathrm{C}_{40}$ prenyl substrates containing ring moieties. Thus, head-to302 head condensation of two $\mathrm{C}_{20}$ isoprenyl molecules may take place prior to attachment to 303 the glycerol unit. These $\mathrm{C}_{20}$ isoprenyl molecules contain an isopropylidene double bond 304 required for such condensation ${ }^{39-40}$, except for the unusual $\mathrm{C}_{20}$ isoprenyl unit with a 305 cyclohexane moiety hypothesized for Thaumarchaeota (FIG. 6). This eliminates the 306 need for an unusual (and experimentally poorly supported) condensation of the two 307 saturated phytanyl chains of archaeol (FIG. 1). This proposed head-to-head 308 condensation of two $\mathrm{C}_{20}$ isoprenyl molecules could be potentially catalyzed by phytoene 309 synthase that converts two GGPP $\mathrm{C}_{20}$ into phytoene $\left(\mathrm{C}_{40}\right)$ by tail-to-tail coupling in the 310 second step in the biosynthesis of carotenoids ${ }^{57}$. Interestingly, homologues of phytoene 311 synthase have been annotated in archaeal genomes (Table S2) of the orders Sulfolobales

312 and Themoproteales of the Crenarchaeota phylum, and in the orders

313 Thermoplasmatales, Methanomicrobiales, Methanobacteriales, Methanosarcinales and

314 Halobacteriales of the Euryarchaeota phylum, but not in any of the available genomes of 315 the Thaumarchaeota phylum. The latter might not be surprising as the intermediate $\mathrm{C}_{20}$ 316 GGGP containing the cyclohexane moiety, as hypothesized in our pathway, does not 317 possess a terminal isopropylidene moiety (FIG. 6).

318 After formation of the GGGP, the second IPP unit is attached to the glycerol moiety.

319 The potential presence of ring moieties before the catalysis mediated by GGGP and 320 DGGGP synthases would again explain the diversification of DGGGP synthases 
321 observed in our study. It would also explain the apparent lack of the DGGGP synthase-

322 coding gene in genomes of the Thaumarchaeota phylum by the presence of a more

323 divergent DGGGP synthase that can accommodate the bulky presence of the unique

324 cyclohexane moiety of the biphytanyl chain. Finally, a second glycerol moiety is

325 attached followed by saturation of the isoprenyl chains and attachment of the

326 headgroup. Considering the alternative pathway presented here, we propose to rename

327 the GGGP and DGGGP synthases as isoprenylglyceryl phosphate (IPGP) synthase and

328 di-isoprenylglyceryl phosphate (DIPGP) synthase, respectively, in order to reflect the

329 the more general nature of these enzymes and their independence with respect to the

330 chain length of their substrate (FIG. 6).

331 The proposed pathway is consistent with the analysis of the sequences of key

332 enzymes of the pathway observed in our study, as well as most of the experimental

333 evidence for the different GDGT biosynthetic steps. Furthermore, the isoprenoid

334 glycerol dialkanol diethers (compounds with $\mathrm{C}_{40}$ isoprenoid chains and ring moieties

335 but only attached to one glycerol group), recently detected in archaeal cultures ${ }^{58-59}$, as

336 well as the biphytane diols detected in the environment ${ }^{60}$, are all products of potential

337 intermediates that fit well with our proposed biosynthetic pathway. Clearly, the steps

338 proposed in our hypothetical biosynthetic scheme require experimental verification

339 using archaeal cultures, specifically of the Thaumarchaeota phylum. Such results,

340 together with further genomic data mining, will shed further light on the unique

341 membrane lipid pathway of the Archaea.

\section{References}

343 1. Woese, C. R., \& Fox, G. E. Phylogenetic structure of the prokaryotic domain: The

344 primary kingdoms. Proc. Nat. Acad Sci. USA 74, 5088-5090 (1977). 
2. DeLong, E. F. \& Pace, N. R. Environmental diversity of Bacteria and Archaea. Syst. Biol. 50, 470-478 (2001).

3. Karner, M. B., DeLong, E. F. \& Karl, D. M. Archaeal dominance in the mesopelagic zone of the Pacific Ocean. Nature 409, 507-510 (2001).

4. Jarrell, K. F., Walters, A. D., Bochiwal, C., Borgia, J. M., Dickinson, T. \& Chong, J. P. Major players on the microbial stage: why archaea are important. Microbiology 157, 919-936 (2011).

5. Wuchter, C., Abbas, B., Coolen, M. J. L., Herfort, L., Timmers, P., Strous, M., van Bleijswijk, J., Teira, E., Herndl, G. J., Middelburg, J. J., Schouten, S., \& Sinninghe Damsté, J. S. Archaeal nitrification in the ocean. Proc. Nat. Acad. Sci. USA 103, 12317-12322 (2006).

6. Koga, Y.\& Morii, H. Biosynthesis of ether-type polar lipids in archaea and evolutionary considerations. Microbiol. Mol. Biol. Rev. 71, 97-120 (2007). An extensive review of the different steps in the archaeal ether lipid biosynthetic pathway and the experimental data supporting this.

7. Kates, M. Biology of halophilic bacteria, Part II. Membrane lipids of extreme halophiles: biosynthesis, function and evolutionary significance. Experientia 49, 1027-1036 (1993).

8. Thompson, D. H., Wong, K. F., Humphry-Baker, R., Wheeler, J. J., Kim, J.-M. \& Rannanavare, S. B. Tetraether bolaform amphiphiles as models of archae-bacterial membrane lipids: Raman spectroscopy, ${ }^{31} \mathrm{P}$ NMR, X-rays scattering, and electron microscopy. J. Am. Chem. Soc. 114, 9035-9042 (1992).

9. Sinninghe Damsté, J. S., Hopmans, E. C., Schouten, S., van Duin, A. C. T. \& Geenevasen, J. A. J. Crenarchaeol: the characteristic core glycerol dibiphytanyl glycerol tetraether membrane lipid of cosmopolitan pelagic crenarchaeota. J. Lipid Res. 43, 1641-1651 (2002).

First description of crenarchaeol structure as a characteristic lipid of marine Thaumarchaeota.

10. Boucher, Y., Kamekura, M., \& Doolittle, W. F. Origins and evolution of isoprenoid lipid biosynthesis in archaea. Mol. Microbiol. 52, 515-527 (2004).

11. Lombard, J., Lopez-Garcia, P., \& Moreira D. Phylogenomic investigation of phospholipid synthesis on Archaea. Archaea 2012, 630910 (2012).

12. Schouten, S, Hopmans, E. C. \& Sinninghe Damsté, J. S. The organic geochemistry of glycerol dialkyl glycerol tetraether lipids: A review. Org. Geochem. 54, 19-61 (2013).

A recent review on the sources, analysis methods, and applications of archaeal ether lipids in organic geochemistry.

13. Woese, C. R., Kandler, O. \& Wheelis, M. L. Towards a natural system of organisms: Proposal for the domains Archaea, Bacteria, and Eucarya. Proc. Natl. Acad. Sci. USA. 87, 4576-4579 (1990). 
14. Gribaldo, S. \& Brochier-Armanet, C. The origin and evolution of Archaea: a state of the art. Philos Trans. R. Soc. Lond. B Biol. Sci. 361, 1007-1022 (2006).

15. Stetter, K.O., Fiala, G., Huber, G., Huber, R. \& Segerer, A. Hyperthemophilic microorganisms. FEMS Microbiol. Rev. 75, 117-124 (1990).

16. Madsen, E. L. Environmental Microbiology: From Genomes to Biogeochemistry. Wiley-Blackwell eds . (2008).

17. Nelson-Sathi, S., Dagan, T., Janssen, A., Steel, M, McInerney, J. O., Deppenmeier, U. \& Martin, W. F. Acquisition of 1,000 eubacterial genes physiologically transformed a methanogen at the origin of Haloarchaea. Proc. Natl. Acad. Sci. USA. doi:10.1073/pnas.1209119109 (2012).

18. Ruepp, A., Graml, W., Santos-Martinez, M. L., Koretke, K. K., Volker, C., Mewes, $\mathrm{H}$. W, et al. The genome sequence of the thermoacidophilic scavenger Thermoplasma acidophilum. Nature 407, 508-5139 (2000).

19. Brochier-Armanet, C., Forterre, P. \& Gribaldo, S. Phylogeny and evolution of the Archaea: one hundred genomes later. Curr. Opin. Microbiol. 14, 274-281 (2011).

20. Elkins, J. G., Podar, M., Graham, D. E., Makarova, K. S., Wolf, Y., Randau, L., Hedlund, B. P., Brochier-Armanet, C., Kunin, V., Anderson, I et al. A korarchaeal genome reveals insights into the evolution of the Archaea. Proc. Natl. Acad. Sci. USA. 105, 8102-8107 (2008).

21. Huber, H., Hohn, M. J., Stetter, K. O., \& Rachel, R. The phylum Nanoarchaeota: present knowledge and future perspectives of a unique form of life. Res. Microbiol. 154, 165-171 (2003).

22. Brochier-Armanet, C., Boussau, B., Gribaldo, S. \& Forterre, P. Mesophilic Crenarchaeota: proposal for a third archaeal phylum, the Thaumarchaeota. Nat. Rev. Microbiol. 6, 245-252 (2008).

\section{Analysis report suggesting the classification of Thaumarchaeota as a separate phylum of the Archaea.}

23. Nunoura, T.,Takaki, Y., Kakuta, J., Nishi, S., Sugahara, J., Kazama, H., Chee, G-J., Hattori, M., Kanai, A., Atomi, H., Takai, K. \& Takami, H. Insights into the evolution of Archaea and eukaryotic protein modifier systems revealed by the genome of a novel archaeal group. Nucleic Acids Res. 39, 3204-3223 (2011).

24. Pester, M., Schleper, C., \& Wagner, M. The Thaumarchaeota: an emerging view of their phylogeny and ecophysiology. Curr. Opin. Microbiol. 14, 300-306 (2011).

25. Koga, Y., \& Morii, H. Recent advances in structural research on ether lipids from Archaea including comparative and physiological aspects. Biosci. Biotechnol. Biochem. 69, 2019-2034 (2005).

26. Koga, Y., Akagawa-Matsushita, M., Ohga, M. \& Nishihara, M. Taxonomic significance of the distribution of component parts of polar ether lipids in methanogens. Syst. Appl. Microbiol. 16, 342-351 (1993). 
27. Langworthy, T.A. Lipids of archaebacteria. In The Bacteria (Woese, C. R. \& Wolfe, R. S., ed.) 459-497 (New York, Academic Press, 1985).

28. Uda, I, Sugai, A., Itoh, Y. H., Itoh, T. Variation in molecular species of polar lipids from Thermoplasma acidophilum depends on growth temperature. Lipids 36, 103105 (2001).

29. Chong, P. L. G. Archaebacterial bipolar tetraether lipids: physico-chemical and membrane properties. Chem. Phys. Lip. 163, 253-65 (2010).

30. Macalady, J. L., Vestling, M. M., Baumler, D., Boekelheide, N., Kaspar, C. W. \& Banfield, J. F. Tetraether-linked membrane monolayers in Ferroplasma spp: a key to survival in acid. Extremophiles 8, 411-419 (2004).

31. Pitcher, A., Hopmans, E. C., Mosier, A. C., Park, S., Rhee, S., Francis, C. A., Schouten, S. \& Sinninghe Damsté, J. S. Core and Intact Polar Glycerol Dibiphytanyl Glycerol Tetraether Lipids of Ammonia-Oxidizing Archaea Enriched from Marine and Estuarine Sediments. Appl. Environ. Microbiol. 77, 3468-3477 (2011).

32. Reysenbach, A. L., Liu, Y., Banta, A. B., Beveridge, T. J., Kirshtein, J. D., Schouten, S., Tivey, M. K., Von Damm, K. \& Voytek, M. A. Isolation of a ubiquitous obligate thermoacidophilic archaeon from deep-sea hydrothermal vents. Nature 442, 444-447 (2006).

33. De Rosa, M., Gambacorta, A., Nicolaus, B., Ross, H. H. M., Grant, W. D. \& Bu'Lock, J. D. An asymmetric archaebacterial diether lipid from alkaliphilic halophiles. J. Gen. Microbiol. 128, 343-348 (1982).

34. Matsumi, R., Atomi, H., Driessen, A. J. \& van der Oost, J. Isoprenoid biosynthesis in Archaea--biochemical and evolutionary implications. Res. Microbiol. 162, 39-52 (2011).

35. Lai, D. Isoprenoid ether lipid biosynthesis in the extremophile, Archaeoglobus fulgidus. University of California, Los Angeles (2009) PhD dissertation.

36. Langworthy, T. A. Turnover of di-O-phytanylglycerol in Thermoplasma. Rev. Infect. Dis. 4, S266 (1982).

37. Nemoto, N., Shida, Y., Shimada, H., Oshima, T., \& Yamagishi, A. Characterization of the precursor of tetraether lipid biosynthesis in the thermoacidophilic archaeon Thermoplasma acidophilum. Extremophiles 7, 235-243 (2003).

38. Poulter, C. D., Aoki, T., \& Daniels, L. Biosynthesis of isoprenoid membranes in the methanogenic archaebacterium Methanospirillum hungatei. J. Am. Chem. Soc. 110, 2620-2624 (1988).

39. Eguchi, T., Takyo, H., Morita, M., Kakinuma, K., \& Koga, Y. Unusual double-bond migration as plausible key reaction on the synthesis of the isoprenoid membrane lipids of methanogenic archaea. J. Chem. Soc. Chem. Commun. 2000, 1545-1546 (2000). 
40. Eguchi, T., Nishimura, Y., \& Kakinuma. Importance of the isopropylene terminal of geranylgeranyl group for the formation of tetraether lipid in methanogenic archaea. Tetrahedron Lett. 44, 3275-3279 (2003).

41. Wang, K. C., \& Ohnuma, S-I. Isoprenyl diphosphate synthases. Biochim. Biophys. Acta. 1529, 33-48 (2000).
A review of the classification, catalytic mechanism, and chain length determination of isoprenyl diphosphate synthases.

42. Wang, K. \& Ohnuma, S. Chain-length determination mechanism of isoprenyl diphosphate synthases and implications for molecular evolution. Trends. Biochem. Sci. 24, 445-451 (1999).

43. Chen, A., \& Poulter, C. D. Purification and characterization of farnesyl diphosphate/geranylgeranyl diphosphate synthase. A thermostable bifunctional enzyme from Methanobacterium thermoautotrophicum. J. Biol. Chem. 268, 11002 11007 (1993).

44. Ogawa, T., Yoshimura, T., \& Hemmi, H. Geranylfarnesyl diphosphate synthase from Methanosarcina mazei: different role, different evolution. Biochem. Biophys. Res. Commun. 393, 16e20 (2010).

45. Peters, K. E., Wlaters C. C., and Moldowan, J. M. The biomarker guide. Cambridge University Press (2007).

46. Edgar, R. C. MUSCLE: a multiple sequence alignment method with reduced time and space complexity. BMC Bioinformatics 5, 113 (2004).

47. Zhang, D. \& Poulter, C. D. Biosynthesis of archaebacterial ether lipids. Formation of ether linkages by prenyltransferases. J. Am. Chem. Soc. 115, 1270-1277 (1993).

48. Payadeh, J., Fujihashi, M., Gillon, W. \& Pai, E. F. The crystal structure of (S)-3-Ogeranylgeranylglyceryl phosphate synthase reveals an ancient fold for an ancient enzyme. J. Biol. Chem 281, 6070-6078 (2006).

\section{First report of the crystal structure of an archaeal geranylgeranylglyceryl phosphate synthase.}

49. Guldan, H., Matysik, F-M., Bocola, M., Sterner, R., \& Babinger, P. Functional assignment of an enzyme that catalyzes the synthesis of an Archaea-type ether lipid in Bacteria. Angew. Chem. Int. Ed. 50, 8188-8191 (2011).

50. Ren, F., Feng, X., Ko, T-P., Huang, C-H., Hu, Y., Chan, H-C., Liu, Y-L., Wang, K., Chen, C-C., Pang, X., He, M., Li, Y., Oldfield, E., \& Guo, R-T. Insights into TIMbarrel prenyl transferase mechanisms: Crystal structures of PcrB from Bacillus subtilis and Staphylococcus aureus. ChemBioChem. 14, 195-199 (2013).

51. Cole, C., Barber, J. D., \& Barton, G. J. The Jpred 3 secondary structure prediction server. Nucleic Acids Res. 35, W197-W201 (2008). 


\section{Competing interest statement} Biol. 56, 564-577 (2007). sediments. Org. Geochem. 43, 50-55 (2012). 1319 (1998).

The authors declare no competing financial interest.

52. Talavera, G., \& Castresana, J. Improvement of phylogenies after removing divergent and ambiguously aligned blocks from protein sequence alignments. Syst.

53. Guindon, S., Dufayard, J. F., Lefort, V., Anisimova, M., Hordijk, W., \& Gascuel O. New algorithms and methods to estimate maximum-likelihood phylogenies: assessing the performance of PhyML 3.0. Syst. Biol. 59, 307-321 (2010).

54. Abascal, F., Zardoya R., \& Posada D. ProtTest: Selection of best-fit models of protein evolution. Bioinformatics 21, 2104-2105 (2005).

55. Letunic, I., \& Bork, P. Interactive Tree Of Life (iTOL): an online tool for phylogenetic tree display and annotation. Bioinformatics 23, 127-128 (2007).

56. Hemmi, H., Shibuya, K., Takahashi, Y., Nakayama, T. \& Nishino, T. J. (S)-2,3-DiO-geranylgeranylglyceryl phosphate synthase from the thermoacidophilic archaeon Sulfolobus solfataricus. Molecular cloning and characterization of a membraneintrinsic prenyltransferase involved in the biosynthesis of archaeal ether-linked membrane lipids. Biol. Chem. 279, 50197-50203 (2004).

57. Klassen, J. L. Phylogenetic and evolutionary patterns in microbial carotenoid biosynthesis are revealed by comparative genomics. Plos One 5, e11257 (2010).

58. Knappy, C.S. \& Keely, B. J. Novel glycerol dialkanol triols in sediments: transformation products of glycerol diphytanyl glycerol tetraether lipids or biosynthetic intermediates? Chem. Commun. 48, 841-843 (2012).

59. Liu, X. -L., Lipp, J. S., Schröder, J. M., Summons, R. E. \& Hinrichs, K. -U. Isoprenoid glycerol dialkanol diethers: A series of novel archaeal lipids in marine

60. Schouten, S., Hoefs, M. J. L., Koopmans, M. P., Bosch, H-J., \& Sinninghe Damsté, J. S. Structural characterization, ocurrence and fate of archaeal ether-bound acyclic and cyclic biphytanes and corresponding diols in sediments. Org. Geochem. 29, 1305- 
531 Figure 1. Current conception of the archaeal lipid biosynthetic pathway (after $\left.{ }^{6}\right)$.

532 The two basic building blocks are the five-carbon compound isopentenyl phosphate and 533 its isomer dimethylallyl diphosphate (DMAPP) are synthesized by the mevalonate 534 pathay in Archaea ${ }^{6}$. DMAPP consecutively condenses with several isopentenyl 535 diphosphate units to form geranylgeranyl diphosphate (GGPP, $\mathrm{C}_{20}$ ) by an isoprenyl 536 diphosphate (IPP) synthase, GGPP synthase. Dihydroxyacetone phosphate (DHAP) is catalyzed to form glycerol-1-phosphate (G1P). The formation of the two ether bonds between G1P and the GGPP units is catalyzed by the geranylgeranylglyceryl phosphate (GGGP) synthase and the digeranylgeranylglyceryl phosphate (DGGGP) synthase.

540 Then, CDP (cytidine-diphosphate)-diglyceride synthase replaces the phosphate group of 541 the unsaturated DGGGP by CDP generating CDP-DGGGP (unsaturated), which is then 542 replaced by a polar headgroup by a CDP-alcohol phosphatidyl transferase ${ }^{34}$. Saturation 543 of the side chains is supposed to be mediated by geranylgeranyl reductases. The 544 formation of GDGTs is thought to involve a head-to-head coupling between the two 545 archaeol lipids followed by internal cyclization to form cyclopentane moieties. The

547 Figure 2. Partial isoprenyl diphosphate (IPP) synthases protein alignment.

548 Alignment of amino acid sequences of putative IPP synthases identified in genomes of different archaeal orders showing a 'bulky' amino acid residue (tyrosine, Y; phenylalanine, F) at the $5^{\text {th }}$ position before the first aspartate (D)-rich motif, indicating that they are short-chain IPP synthases elongating the isoprenoid chain up to 20 carbon atoms.

Sequences were aligned by MUSCLE (multiple sequence comparison by logexpectation; REF 46). Species detailed in the alignment: S.acidocaldarius (Sulfolobus acidocaldarius), D.kamchatkensis (Desulfurococcus kamchatkensis), A.fulgidus (Archaeoglobus fulgidus), M.smithii (Methanobrevibacter smithii), M.thermophila (Methanosaeta thermophila), M.maripaludis (Methanococcus maripaludis), M.hungatei (Methanospirillum hungatei), T.acidophilum (Thermoplasma acidophilum), N.maritimus (Nitrosopumilus maritimus). Accession numbers are listed in Table S1.

560 Figure 3. Partial geranylgeranylglyceryl phosphate (GGGP) synthase protein 561 alignment.

562 Annotated putative GGGP synthases of representatives of different archaeal orders are 563 aligned and compared to the GGGP synthase protein of A. fulgidus which crystalline structure has been previously determined ${ }^{48}$. The black star indicates the position of the amino acid residue corresponding to the $\mathrm{W}_{99}$ position of $A$. fulgidus. The red star indicates the position containing a tryptophan (W) in the thaumarchaeotal sequences as discussed in the text. The chain-length determination area is arbitrary and indicated for clarification purposes. Amino acids: W (tryptophan), A (alanine), Y (tyrosine), E (glutamine). Location of $\alpha$-helixes according to the $A$. fulgidus crystal structure ${ }^{48}$ in the partial sequence is indicated above the alignment. Black boxes surrounding amino acid 
571 sequences in the alignment correspond to $\alpha$-helix prediction by the Jpred 3 server ${ }^{51}$.

572 Cluster 1, 2A and 2B correspond to the clusters also indicated in Figure 4.

573 Sequences were aligned by MUSCLE ${ }^{46}$. Species detailed in the alignment: A.fulgidus 574 (Archaeoglobus fulgidus), H.salinarium (Halobacterium salinarium), M.limicola 575 (Methanoplanus limnicola), N.maritimus (Nitrosopumilus maritimus), T.neutrophilus 576 (Thermoproteus neutrophilus), A.pernix (Aeropyrum pernix), S.acidocaldarius 577 (Sulfolobus acidocaldarius), D.kamchatkensis (Desulfurococcus kamchatkensis), 578 M.maripaludis (Methanococcus maripaludis), T.litoralis (Thermococcus litoralis), 579 M.marburgensis (Methanothermobacter marburgensis), T.volcanicum (Thermoplasma 580 volcanicum), unc. Euryarchaeota A.boonei (unclassified euryarchaeota 581 Aciduliprofundum boonei), M.thermophila (Methanosaeta thermophila), PcrB Bs (PcrB 582 protein of Bacillus subtilis; accession number YP_007532597.1).

583 Figure 4. Maximum likelihood tree based on the protein sequences of archaeal 584 putative geranylgeranylglyceryl phosphate (GGGP) synthases.

585 Cluster 1 consists on divergent putative GGGP synthases of the euryarchaeotal orders 586 Archaeoglobales, Methanomicrobiales and Halobacteriales. The second cluster is 587 subdivided into cluster 2A, which includes GGGP synthases of the Thaumarchaeota and 588 the Crenarchaeota, and cluster 2B, which includes the remaining GGGP synthases of 589 other euryarchaeotal groups.

590 The scale bar represents number of substitutions per site. Abbreviations: THAUM: 591 Thaumarchaeota; CREN: Crenarchaeota; EURY: Euryarchaeota. The colored symbols 592 indicate the presence of the various membrane lipids (Table 1); Archaeol (dark blue 593 circle); extended archaeol (light blue pentagon); GDGT-0 (red rectangle; GDGT-1-4 594 (yellow triangle); GDGT-5-8 (purple hexagon); Crenarchaeol (green cross). Sequences 595 were aligned using MUSCLE ${ }^{46}$. Alignment was trimmed in Gblocks $0.91 \mathrm{~b}$ with relaxed 596 parameters $^{52}$ and manually curated. Phylogenetic tree was computed by PHYML v3.0 ${ }^{53}$ 597 using the LG model plus gamma distribution and invariant site $(\mathrm{LG}+\mathrm{G}+\mathrm{I})$ indicated by 598 ProtTest $2.4^{54}$. Branch support was calculated with the approximate likelihood ratio test 599 (aLRT) and indicated on the branches (color code in the nodes: red ( $\geq 90 \%)$, blue $600(\geq 70 \%,<90 \%)$ and green $(\geq 50 \%,<70 \%)$, less than $50 \%$ is not shown). Trees were 601 edited in iTOL $^{55}$.

602 Figure 5. Maximum likelihood tree based on the protein sequences of archaeal 603 putative digeranylgeranylglyceryl phosphate (DGGGP) synthases and 604 thaumarchaeotal prenyltransferases.

605 Star symbols indicate Sulfolobus solfataricus DGGGP synthase (AAK40896), and S. 606 solfataricus UbiA-1 (AAK4048.1) previously tested ${ }^{56}$. †Ca. Caldiarchaeum 607 subterraneum Aigarchaeota phylum ${ }^{23}$. For explanation of symbols and legends see FIG.

608 4. The tree was computed as described in the legend of FIG.4.

609 Figure 6. Alternative archaeal lipid biosynthesis scheme based on a multiple-key, 610 multiple-lock mechanism.

611 Isoprenyl diphosphate synthases generate $C_{20}$ isoprenoid units with or without ring 612 moieties during their catalysis. Triangles indicate the introduction of the cyclohexane 613 moiety in the precursor of crenarchaeol in Thaumarchaeota. Condensation of two $\mathrm{C}_{20}$ 
614 isoprenoid units produce a variety of $\mathrm{C}_{40}$ substrates (multiple-keys) that are then used as 615 substrate by isoprenylglyceryl phosphate (IPGP) (*) and di-isoprenylglyceryl phosphate 616 (DIPGP) $(* *)$ synthases (multiple-locks), followed by the attachment of the $2^{\text {nd }}$ glycerol 617 moiety, saturation of the isoprenoid chains, and final attachment of the headgroups.

618 Note that the hydrogenation step is indicated here after assembly of the GDGT but 619 potentially could also occur prior to attachment of IPGP to the glycerol moiety. The 620 formation of the cyclohexane moiety in Thaumarchaeota is indicated here during the 621 formation of the $\mathrm{C}_{20}$ isoprenoid but this leads to an intermediate without a terminal 622 double bond potentially inhibiting head-to-head-coupling of $\mathrm{C}_{20}$ isoprenyl units.

624 Table 1. Distribution of archaeal membrane lipids in different orders of the 625 Euryarchaeota, Crenarchaeota and Thaumarchaeota.

626

627 Table S1. Isoprenyl diphosphate synthases in archaeal genomes.

628 Table S2. Squalene/phytoene synthase homologues annotated in archaeal genomes. 


\section{Glossary}

631 Isoprenoid: Group of natural products with diverse structures composed of various

632 numbers of isopentenyl $\left(\mathrm{C}_{5}\right)$ pyrophosphate (IPP) units

633 Phytanyl: Saturated chain composed of 4 head-to-tail linked isoprene units $\left(\mathrm{C}_{20}\right.$

634 isoprenoid).

635 Biphytanyl: Molecule composed of two head-to-head condensed phytanyl units $\left(\mathrm{C}_{40}\right.$ 636 isoprenoid).

637 Hyperthermophile: Organism that has an optimal growth temperature of at least $80^{\circ} \mathrm{C}$.

638 Thermoacidophile: Combination of thermophile and acidophile (thrive under highly

639 acidic conditions, around $\mathrm{pH} 2.0$ or below), microorganisms that thrive in acid, sulfur

640 rich, and high temperature environments.

641 Halophile: Extremophilic organism that thrives at high concentrations of salt.

642 Methanogen: Archaeon that produces methane under anoxic conditions.

643 Horizontal gene transfer: Transfer of genetic material between different species of 644 microorganisms in which the acquired genes are transmitted to the next generation as 645 the cell divides.

646 Mesophile: Organism that grows at a moderate temperature, typically between 20 and $64745^{\circ} \mathrm{C}$.

648 Diphosphate: Also known as pyrophosphate, ester containing two phosphate groups.

649 Allylic: Double bond at the terminal position of a carbon chain.

650 Prenyltransferases: Enzymes that transfer (iso)prenyl moieties to acceptor molecules.

651 Head-to-head condensation: Coupling of two isoprenyl units at position C1 of both 652 units.

653 Isopropylidene: An isopropyl moiety with a terminal double bond.

654 Squalene: Biochemical precursor of the steroid and triterpenoid families. Synthesized 655 by tail-to-tail condensation of farnesyl pyrophosphate $\left(C_{15}\right)$ by squalene synthase.

656 Phytol: acyclic diterpene (terpene consist of two or more isoprene $\mathrm{C}_{5} \mathrm{H}_{8}$ units) alcohol.

657 Geranylgeraniol: diterpenoid alcohol (3,7,11,15-tetramethyl-2,6,10,14-hexadecatraen658 1-ol).

659 Paralogues: Genes that derive from the same ancestral gene. 


\section{Online Summary}

661

662

663

664

665

666

667

668

669

670

671

672

673

674

675

676

677

678

679

680

681

682

683

684

685

686

687

688

689

690

691

692

693

694

695

696

697

698

- Archaea were initially thought to be confined in extreme environments but now they are known to occur ubiquitously in nature and be important players in global biogeochemical cycles. Archaea are characterized by their unique membrane lipids containing isoprene units linked to the glycerol backbone by ether bonds (archaeol, $\mathrm{C}_{20}$, in a bilayer and glycerol diakyl glycerol tetraether, GDGT, $\mathrm{C}_{40}$ in a monolayer).

- Comparison of the phylogenetic composition of Archaea with the distribution of membrane ether lipid shows that most lipids are not specific for a certain phylogenetic group. Only the GDGT crenarchaeol, containing four cyclopentane moieties and a cyclohexane moiety, is considered to be characteristic of the Thaumarchaeota, suggesting that the biosynthesis of the cyclohexane moiety is unique within this phylum.

- The current conception of the archaeal membrane ether lipid biosynthetic pathway involves the condensation of units of isopentenyl diphosphate to form geranylgeranyl (GGPP, $\mathrm{C}_{20}$ ) by a GGPP synthase. The formation of the two ether bonds is catalyzed by the geranylgeranylglyceryl phosphate (GGGP) synthase and the digeranylgeranylglyceryl phosphate (DGGGP) synthase. The formation of GDGTs is thought to involve a head-to-head coupling between the two archaeol lipids followed by internal cyclization to form cyclopentane moieties. The latter reactions are highly unusual and the enzymes involved are unknown.

- The analysis of the amino acid sequence of most of the archaeal GGGP synthases suggest that they could accommodate substrates $>\mathrm{C}_{20}$ and with rings already present.

- The synthesis of the unique cyclohexane moiety-containing GDGT crenarchaeol by Thaumarchaeota might explain the inability to annotate DGGGP synthases in thaumarchaeotal genomes, as a yet-unknown or divergent DGGGP synthase would be required to accommodate the isoprenyl chain containing the 'bulky' cyclohexane moiety.

- An alternative archaeal lipid biosynthetic pathway pathway is presented based on a multiple-key, multiple-lock mechanism for which multiple keys with different configurations due to the presence of rings, would need to accommodate and specifically interact at the molecular level with different locks (isoprenylglyceryl phosphate, IPGP and di-isoprenylglyceryl phosphate, DIPGP synthases). This pathway is consistent with most of the phylogenetic relationships observed in our study as well as with most of the experimental evidence for the different GDGT biosynthetic steps, and it is supported by possible intermediates previously described. 


\section{Author biographies}

\section{Dr. Laura Villanueva}

701 Research Scientist in the Department of Marine Organic Biogeochemistry at the Royal

702 Netherlands Institute for Sea Research (NIOZ). Her research interests are in the field of 703 molecular geomicrobiology: Molecular evolution of lipid synthetic pathways, 704 development of genetic tools to detect lipid biomarker-producing microorganisms, and 705 microbial ecology of marine microorganisms especially those involved in the nitrogen 706 cycle.

Prof. Dr. Ir. Jaap S. Sinninghe Damsté

709 Professor of Organic Geochemistry at Utrecht University (Faculty of Geosciences) and 710 head of the Department of Marine Organic Biogeochemistry at NIOZ. He has been 711 working for $>25$ years on the occurrence and characterization of lipids in microbes 712 (bacteria, archaea, algae) and their application as biomarkers in oceanography, 713 geochemistry, and palaeoclimatology.

\section{Prof. Dr. Ir. Stefan Schouten}

717 Professor in Molecular Palaeontology in Utrecht University (Faculty of Geosciences) 718 and Senior Research Scientist at NIOZ. His research interest is the organic 719 biogeochemistry of marine sediments, i.e. the reconstruction of present and past 720 microbial communities, biosynthetic pathways, biogeochemical cycles, environments 721 and climates by structural and stable isotopic analysis of organic compounds in 722 microorganisms, marine waters and sediments. 
Table 1. Distribution of archaeal membrane lipids in orders of the Euryarchaeota, Crenarchaeota and Thaumarchaeota phyla.

\begin{tabular}{|c|c|c|c|c|c|c|c|c|c|}
\hline \multirow{2}{*}{$\begin{array}{l}\text { ARCHAEAL LIPIDS } \\
\text { PHYLOGENY }\end{array}$} & \multirow[b]{2}{*}{ 营 } & \multirow[b]{2}{*}{ 竞 } & \multirow[b]{2}{*}{ 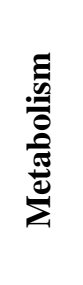 } & \multirow[b]{2}{*}{ "orapysy } & \multirow{2}{*}{ 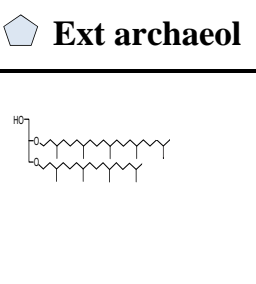 } & \multirow[t]{2}{*}{ GDGT-0 } & \multirow{2}{*}{ 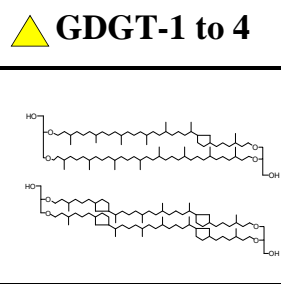 } & \multirow{2}{*}{ GDGT-5 to 8} & \multirow{2}{*}{ Crenarchaeol } \\
\hline & & & & & & & & & \\
\hline \multicolumn{10}{|l|}{ Euryarchaeota } \\
\hline Halobacteriales & $\mathrm{M}$ & N/Al & $\mathrm{H}$ & $\sqrt{ }$ & $\sqrt{ }$ & & & & \\
\hline Methanosarcinales & $\mathrm{M}$ & $\mathrm{N}$ & Met & $\sqrt{ }$ & & & & & \\
\hline Methanopyrales & $\mathrm{H}$ & $\mathrm{N}$ & Met & $\sqrt{ }$ & & & & & \\
\hline Methanococcales & $\mathrm{M} / \mathrm{T}$ & N/Al & Met & $\sqrt{ }$ & & & & & \\
\hline Thermococcales & $\mathrm{T} / \mathrm{H}$ & $\mathrm{N}$ & $\mathrm{S}$ & $\sqrt{ }$ & & $\sqrt{ }$ & & & \\
\hline Methanobacteriales & $\mathrm{M} / \mathrm{T}$ & $\mathrm{N}$ & Met & $\sqrt{ }$ & & $\sqrt{ }$ & & & \\
\hline Archaeoglobales & $\mathrm{M} / \mathrm{T}$ & $\mathrm{Al}$ & $\mathrm{S}$ & & & $\sqrt{ }$ & & & \\
\hline Methanomicrobiales & $\mathrm{M}$ & $\mathrm{N}$ & Met & & & $\sqrt{ }$ & & & \\
\hline Thermoplasmatales* & $\mathrm{M} / \mathrm{T}$ & Ac & $\mathrm{S}$ & & & $\sqrt{ }$ & $\sqrt{ }$ & $\sqrt{ }$ & \\
\hline \multicolumn{10}{|l|}{ Crenarchaeota } \\
\hline Thermoproteales & $\mathrm{T} / \mathrm{H}$ & N/Ac & $\mathrm{S}$ & & & $\sqrt{ }$ & $\sqrt{ }$ & $\sqrt{ }$ & \\
\hline Sulfolobales & $\mathrm{T} / \mathrm{H}$ & Ac & $\mathrm{S}$ & & & $\sqrt{ }$ & $\sqrt{ }$ & $\sqrt{ }$ & \\
\hline Acidilobales & $\mathrm{H}$ & Ac & Org & & & $\sqrt{ }$ & $\sqrt{ }$ & $\sqrt{ }$ & \\
\hline Desulfurococcales & $\mathrm{H}$ & $\mathrm{N}$ & $\mathrm{S}$ & & & $\sqrt{ }$ & $\sqrt{ }$ & & \\
\hline \multicolumn{10}{|l|}{ Thaumarchaeota } \\
\hline Cenarchaeales & & & & & & $\sqrt{ }$ & $\sqrt{ }$ & & $\sqrt{ }$ \\
\hline Nitrosopumilales & & & & & & $\sqrt{ }$ & $\sqrt{ }$ & & $\sqrt{ }$ \\
\hline Nitrososphaerales & & & & & & $\sqrt{ }$ & $\sqrt{ }$ & & $\sqrt{ }$ \\
\hline
\end{tabular}

* DHVE-2 cluster (Aciduliprofundum boonei), closely related to the Thermoplasmatales order synthesize GDGT-0, GDGT-1/4 (REF 30). Temperature: M (Mesophile, 20$45^{\circ} \mathrm{C}$ ); $\mathrm{T}$ (Thermophile, $45-80^{\circ} \mathrm{C}$ ); $\mathrm{H}$ (Hyperthermophile, $>80^{\circ} \mathrm{C}$ ). $\mathrm{pH}$ : N (Neutrophile, 5-8); Al (Alkalophile, $>8$ ); Ac (Acidophile, <5). Metabolism: $\mathrm{H}$ (Heterotrophy); Met (Methanogenesis); S (sulfur dependent); Nit (Nitrifier); Org (Organotroph). Archaeal membrane lipids distribution information from Schouten et al. ${ }^{12}$. 
Table S1. Isoprenyl diphosphate (IPP) synthases in archaeal genomes.

\begin{tabular}{|c|c|c|c|c|c|c|}
\hline Phylum & Order & Genus, species & $\begin{array}{l}\text { Short-chain } \\
\text { IPP synthase }{ }^{\ddagger}\end{array}$ & $\begin{array}{c}\text { Amino } \\
\text { acid }^{\dagger}\end{array}$ & $\begin{array}{l}\text { Long-chain } \\
\text { IPP synthase }{ }^{\ddagger}\end{array}$ & $\begin{array}{l}\text { Amino } \\
\text { acid }^{\dagger}\end{array}$ \\
\hline Crenarchaeota & Sulfolobales & Sulfolobus acidocaldarius & YP_254812.1 & $\mathrm{F}$ & YP_255648.1 & $\mathrm{S}$ \\
\hline Crenarchaeota & Sulfolobales & Sulfolobus solfataricus & NP 341633.1 & $\mathrm{~F}$ & NP 343706.1 & $\mathrm{~A}$ \\
\hline Crenarchaeota & Sulfolobales & Sulfolobus tokodaii & NP_378047.1 & $\mathrm{F}$ & NP 376371.1 & $\mathrm{~A}$ \\
\hline Crenarchaeota & Thermoproteales & Pyrobaculum aerophilum & NP 559016.1 & $\mathrm{Y}$ & NP 560635.1 & $\mathrm{V}$ \\
\hline Crenarchaeota & Thermoproteales & Thermoproteus neutrophilus & YP_001793568.1 & $\mathrm{Y}$ & YP_001794908 & $\mathrm{V}$ \\
\hline Crenarchaeota & Thermoproteales & Pyrobaculum islandicum & YP_930716 & $\mathrm{Y}$ & YP_930070.1 & $\mathrm{V}$ \\
\hline Crenarchaeota & Thermoproteales & Caldivirga maquilensis & YP_001540467.1 & $\mathrm{Y}$ & YP_001540335.1 & A \\
\hline Crenarchaeota & Desulfurococcales & Aeropyrum pernix* & BAA88983.1 & $\mathrm{F}$ & & \\
\hline Crenarchaeota & Desulfurococcales & $\begin{array}{l}\text { Desulfurococcus } \\
\text { kamchatkensis }\end{array}$ & YP_002429148.1 & $\mathrm{Y}$ & & \\
\hline Crenarchaeota & Desulfurococcales & Ignicoccus hospitalis & YP_001435752.1 & $\mathrm{F}$ & YP_001434928.1 & $\mathrm{S}$ \\
\hline Crenarchaeota & Desulfurococcales & Staphylothermus marinus & YP_001040510.1 & $\mathrm{Y}$ & & \\
\hline Crenarchaeota & Acidilobales & Acidilobus saccharovorans & YP_003815770.1 & $\mathrm{F}$ & YP 003815825 & A \\
\hline Euryarchaeota & Thermoplasmatales & Thermoplasma volcanicum & NP_110781.1 & $\mathrm{Y}$ & NP 111576.1 & $\mathrm{~A}$ \\
\hline Euryarchaeota & Thermoplasmatales & Thermoplasma acidophilum & NP_394768.1 & $\mathrm{Y}$ & NP_393914 & $\mathrm{A}$ \\
\hline Euryarchaeota & Thermoplasmatales & Ferroplasma acidarmanus & $\mathrm{ZP}=05571075.1$ & $\mathrm{~F}$ & $\mathrm{ZP}=05570405.1$ & $\mathrm{~A}$ \\
\hline Euryarchaeota & Thermoplasmatales & Acididuliprofundum boonei & ZP_04875656.1 & $\mathrm{Y}$ & ZP_04875510.1 & $\mathrm{Y}$ \\
\hline Euryarchaeota & Thermococcales & Thermococcus sp. AM4 & YP_002582296.1 & $\mathrm{Y}$ & YP_002581574.1 & A \\
\hline Euryarchaeota & Thermococcales & Pyrococcus horikoshii & NP_142981.1 & $\mathrm{Y}$ & & \\
\hline Euryarchaeota & Methanobacteriales & Methanobrevibacter smithii & ZP 05975848.2 & $\mathrm{~F}$ & & \\
\hline Euryarchaeota & Methanobacteriales & Methanobacterium & YP_004520238.1 & $\mathrm{F}$ & & \\
\hline Euryarchaeota & Methanobacteriales & $\begin{array}{l}\text { Methanothermobacter } \\
\text { marburgensis }\end{array}$ & YP_003849447.1 & $\mathrm{F}$ & & \\
\hline Euryarchaeota & Methanopyrales & Methanopyrus kandleri & NP_614058 & $\mathrm{F}$ & & \\
\hline Euryarchaeota & Methanococcales & Methanococcus maripadulis & NP_987165.1 & $\mathrm{Y}$ & & \\
\hline
\end{tabular}




\begin{tabular}{|c|c|c|c|c|c|c|}
\hline Euryarchaeota & Methanococcales & $\begin{array}{l}\text { Methanocaldococcus sp. } \\
\text { FS406 }\end{array}$ & YP_003458715.1 & Y & & \\
\hline Euryarchaeota & Methanosarcinales & Methanosaeta thermophila & YP_842903.1 & $\mathrm{F}$ & YP_842784.1 & $\mathrm{A}$ \\
\hline Euryarchaeota & Methanosarcinales & Methanosarcina barkeri & YP 304956.1 & $\mathrm{~F}$ & YP 303957 & $\mathrm{~A}$ \\
\hline Euryarchaeota & Methanosarcinales & Methanosarcina mazei & NP_633791.1 & $\mathrm{F}$ & NP_632813 & $\mathrm{A}$ \\
\hline Euryarchaeota & Methanomicrobiales & Methanospirillum hungatei & YP 504297.1 & $\mathrm{~F}$ & & \\
\hline Euryarchaeota & Methanomicrobiales & Methanoplanus limicola & ZP_09700978.1 & $\mathrm{F}$ & & \\
\hline Euryarchaeota & Archaeoglobales & Archaeoglobus fulgidus & AAD26851.1 & $\mathrm{F}$ & NP 070380.1 & $\mathrm{~A}$ \\
\hline Euryarchaeota & Archaeoglobales & Archaeoglobus veneficus & YP_004341338.1 & $\mathrm{F}$ & YP_004340873 & $\mathrm{A}$ \\
\hline Euryarchaeota & Archaeoglobales & Ferroglobus placidus & YP_003435928.1 & $\mathrm{F}$ & YP_003435725.1 & A \\
\hline Euryarchaeota & Halobacteriales & $\begin{array}{l}\text { Haladaptatus } \\
\text { paucihalophilus }\end{array}$ & ZP_08044024.1 & $\mathrm{F}$ & ZP_08042560 & $\mathrm{A}$ \\
\hline Euryarchaeota & Halobacteriales & Haloarcula hispanica & YP 004795446.1 & $\mathrm{~F}$ & YP 004795026 & A \\
\hline Euryarchaeota & Halobacteriales & Halomicrobium mukohataei & YP_003178421.1 & $\mathrm{F}$ & YP_003177096 & $\mathrm{A}$ \\
\hline Euryarchaeota & Halobacteriales & Natronomonas pharaonis** & YP 327492.1 & $\mathrm{F}$ & YP_325962 & A \\
\hline Euryarchaeota & Halobacteriales & Halobacterium sp. NRC-1 & AAG19532.1 & $\mathrm{F}$ & NP_ 280810 & A \\
\hline Thaumarchaeota & Cenarchaeales & Cenarchaeum symbiosum & YP_876540 & $\mathrm{F}$ & YP_876597.1 & $\mathrm{E}$ \\
\hline Thaumarchaeota & Nitrosopumilales & Ca. Nitrosoarchaeum limnia & ZP_08257374 & $\mathrm{F}$ & $\mathrm{ZP} \quad 08257400.1$ & $\mathrm{E}$ \\
\hline Thaumarchaeota & Nitrosopumilales & $\begin{array}{l}\text { Ca. Nitrosoarchaeum } \\
\text { koreensis MY1 }\end{array}$ & ZP_08667311 & $\mathrm{F}$ & ZP_08667284.1 & $\mathrm{E}$ \\
\hline Thaumarchaeota & Nitrosopumilales & $\begin{array}{l}\text { Ca. Nitrosopumilus salaria } \\
\text { BD31 }\end{array}$ & ZP_10118543 & $\mathrm{F}$ & ZP_10118596.1 & $\mathrm{E}$ \\
\hline Thaumarchaeota & Nitrosopumilales & Nitrosopumilus maritimus & YP_001581646 & $\mathrm{F}$ & YP_001581621.1 & $\mathrm{E}$ \\
\hline Thaumarchaeota & Nitrososphaerales & $\begin{array}{l}\text { Ca. Nitrososphaera } \\
\text { gargensis }\end{array}$ & YP_006862746 & $\mathrm{F}$ & YP_006861760 & $\mathrm{E}$ \\
\hline
\end{tabular}

Short and Long-chain IPP synthases are defined in the text. ${ }^{\mp} \mathrm{NCBI}$ accession number. $\uparrow$ Amino acid residue in the $5^{\text {th }}$ position before the first aspartate-rich motif.

* farnesylgeranyl diphosphate (FGPP) synthase of Aeropyrum pernix is involved in a pathway that only produces $\mathrm{C}_{25}-\mathrm{C}_{25}$ diether lipids. It has been previously suggested that this FGPP synthase has evolved from an ancestral IPP synthase of Desulfurococcales (Tabichana et al., 2000). **bifunctional geranyl/farnesylgeranyl diphosphate synthase $\left(\mathrm{C}_{20}\right.$ and $\mathrm{C}_{25}$, respectively) described in the Halobacteriales Natronomonas pharaonis (Falb et al., 2005). 
Table S2. Squalene/phytoene synthase homologues annotated in archaeal genomes.

\begin{tabular}{llll}
\hline Phylum & Order & Genus species & Accession number \\
\hline Crenarchaeota & Sulfolobales & Sulfolobus acidocaldarius & YP_256333.1 \\
\hline Crenarchaeota & Sulfolobales & Sulfolobus islandicus & YP_002839942.1 \\
\hline Crenarchaeota & Sulfolobales & Sulfolobus solfataricus & NP_344224.1 \\
\hline Crenarchaeota & Sulfolobales & Metallosphaera yellowstonensis & WP_009069731.1 \\
\hline Crenarchaeota & Sulfolobales & Metallophaera cuprina & YP_004409628.1 \\
\hline Crenarchaeota & Sulfolobales & Metallophaera sedula & YP_001191163.1 \\
\hline Crenarchaeota & Thermoproteales & Pyrobaculum oguniense & YP_005260591.1 \\
\hline Crenarchaeota & Thermoproteales & Pyrobaculum arsenaticum & YP_001152662.1 \\
\hline Euryarchaeota & Thermoplasmatales & Picrophilus torridus & AAT_44120.1 \\
\hline Euryarchaeota & Methanobacteriales & Methanobacterium sp. & WP_008515272.1 \\
\hline Euryarchaeota & Methanobacteriales & Methanothermobacter thermoautotrophicus & NP_276914.1 \\
\hline Euryarchaeota & Methanomicrobiales & Methanoculleus marisnigri & YP_001046034.1 \\
\hline Euryarchaeota & Methanosarcinales & Methanosalsum zhilinae & YP_004616025.1 \\
\hline Euryarchaeota & Halobacteriales $\dagger$ & Natrialba madadii & YP_003482007.1 \\
\hline Euryarchaeota & Halobacteriales & Natronobacterium gregoryi & YP_007177834.1 \\
\hline Euryarchaeota & Halobacteriales & Halobacterium sp. & NP_280284.1 \\
\hline Euryarchaeota & Halobacteriales & Haloferax prahovense & WP_0080095376.1 \\
\hline Euryarchaeota & Halobacteriales & Haloquadratum walsbyi & YP_658569.1 \\
\hline Euryarchaeota & Halobacteriales & Haloarcula marismortui & YP_136629.1 \\
\hline
\end{tabular}

Phytoene/squalene synthase are defined as tail-to-tail isoprenyl diphosphate synthases. Squalene and phytoene synthases catalyze the condensation of two $\mathrm{C}_{15}$ (farnesyl) and $\mathrm{C}_{20}$ (geranylgeranyl) isoprenyl diphosphates, respectively. $\dagger$ Halobacteriales: phytoene/squalene synthases are commonly found in members of the Halobacteriales order and here we just list some of them. They are believed to be involved in the formation of rhodopsins formed by halophilic Archaea (Peck et al., 2002). 


\section{References Supplementary Tables:}

Falb, M., Pfeiffer, F., Palm, P., Rodewald, K., Hickmann, V., Tittor, J. \& Oesterhelt, D. Living with two extremes: conclusions from the genome sequence of Natronomonas pharaonis. Genome Res. 15, 1336-1343 (2005).

Peck, R.F., Johnson, E.A., \& Krebs, M.P. Identification of a lycopene beta-cyclase required for bacteriorhodopsin biogenesis in the archaeon Halobacterium salinarum. J. Bacteriol. 184, 2889-2897 (2002).

Tachibana, A., Yano, Y., Otani, S., Nomura, N., Sako, Y.\& Taniguchi, M. Novel prenyltransferase gene encoding farnesylgeranyl diphosphate synthase from a hyperthermophilic archaeon, Aeropyrum pernix. Molecular evolution with alteration in product specificity. Eur. J. Biochem. 267, 321-328 (2000). 
Figure 1.

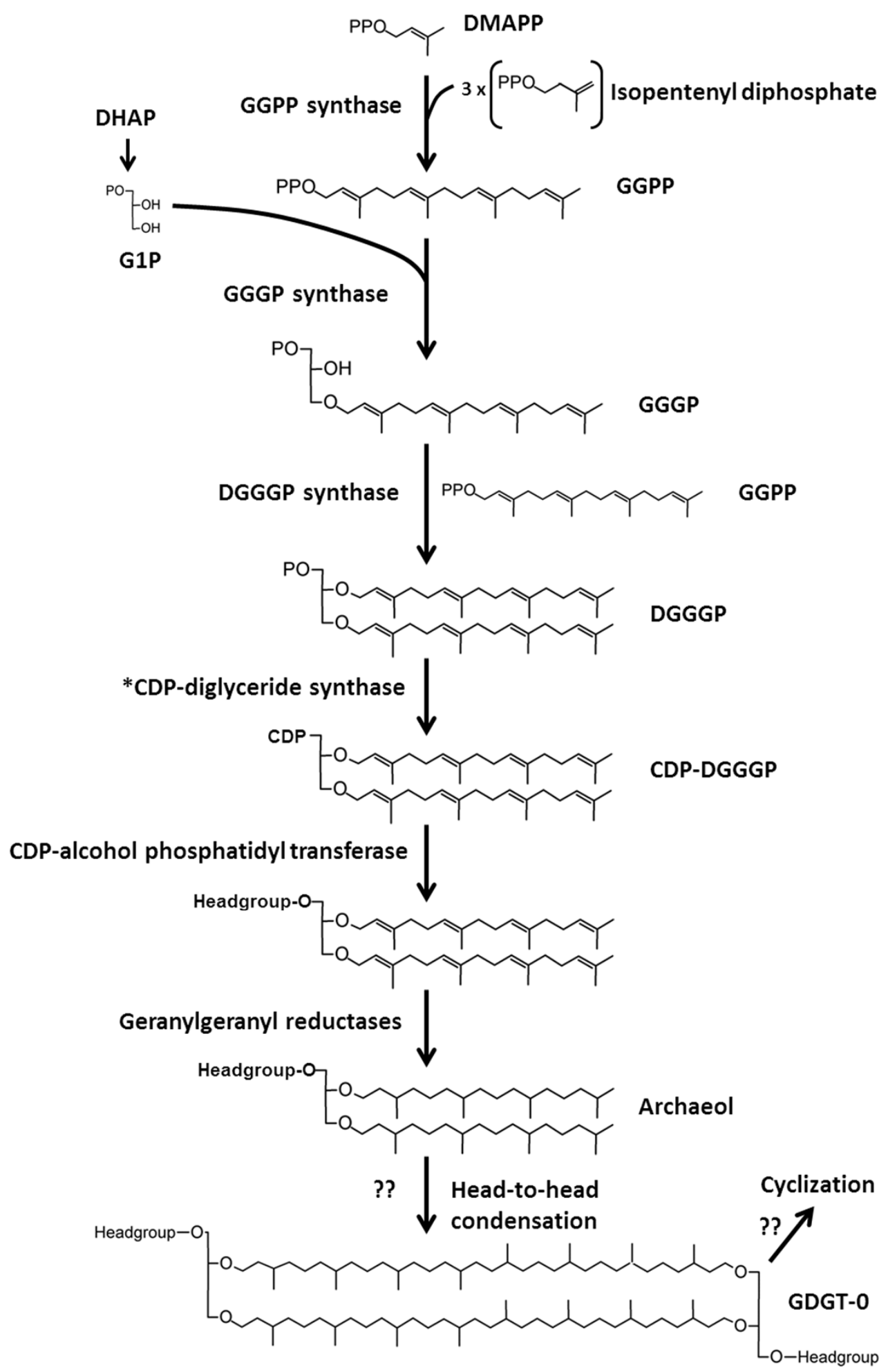


Figure 2

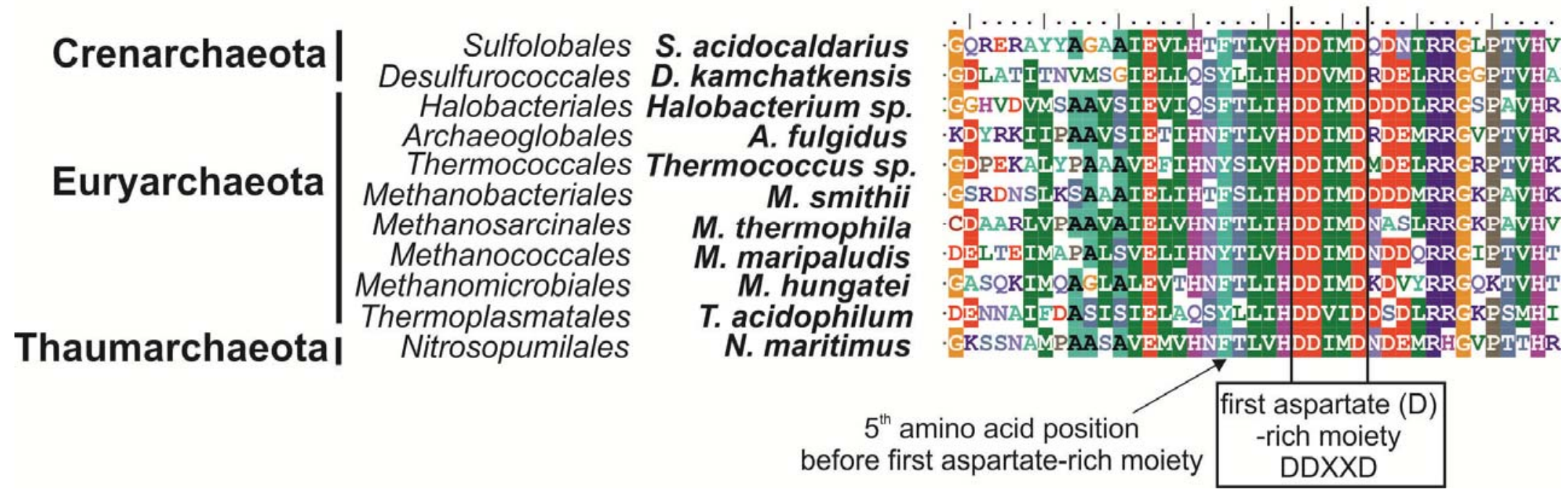


Figure 3

W" (Tryptophan) of A. fulgidus

Binding site of the
$\mathrm{Y}^{165} / \mathrm{E}^{167}$

Conserved residues

that interact with $\mathrm{G} 1 \mathrm{P}$

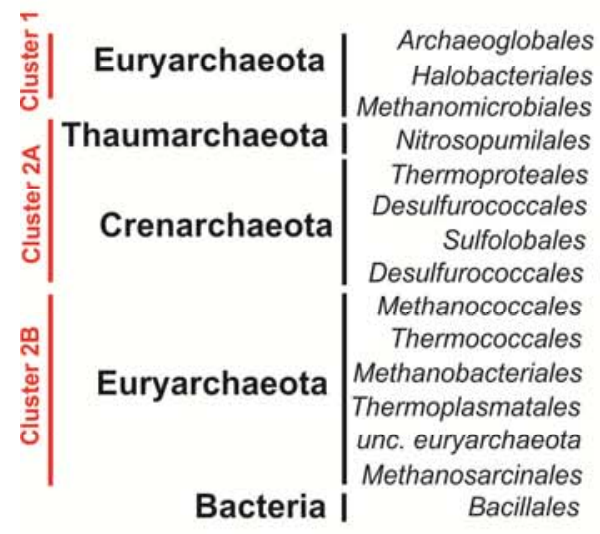

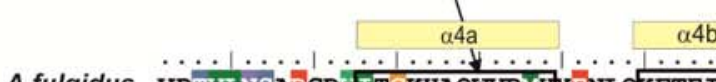

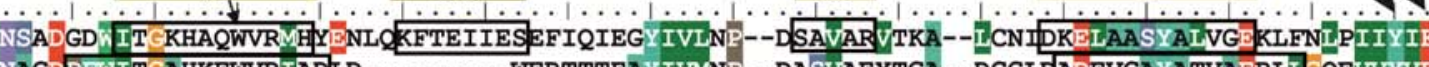

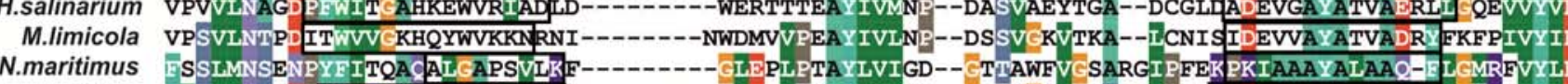

N.marimus
Theutrophilus

A.pernix
ALTVMNSD

S.acidocaldarius

M.maripaludis

T.litoralis

VILND DLYYIVGAQIVAAPIIKR PYYLQVOVIAAPLIRK

I------

GLPVLPTAYLIIGH--GGMACHICRARVI

YDNEITIVSYSLAAN-Z MEMKYIYI

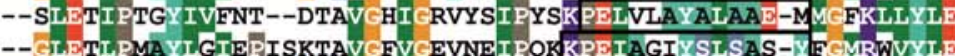

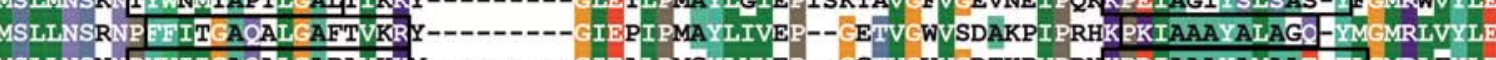

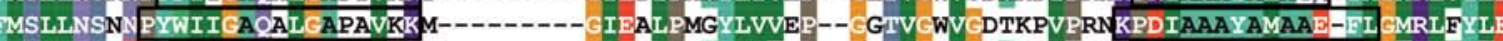

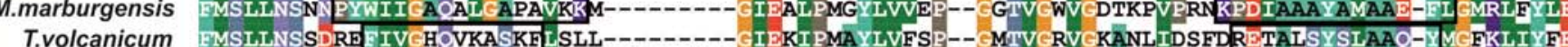

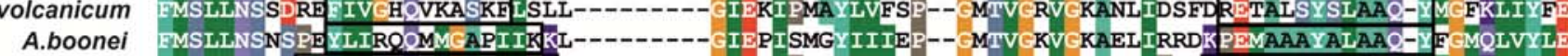

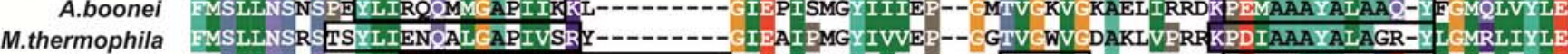

PcrB Bs

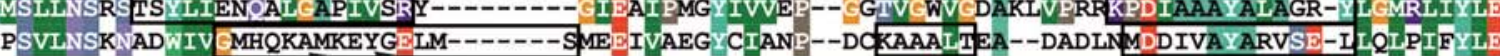$$
\text { ain-1 }
$$

chain-length $A_{100} \& Y_{104}$ residues

area

PcrB B. subtilis 
Figure 4

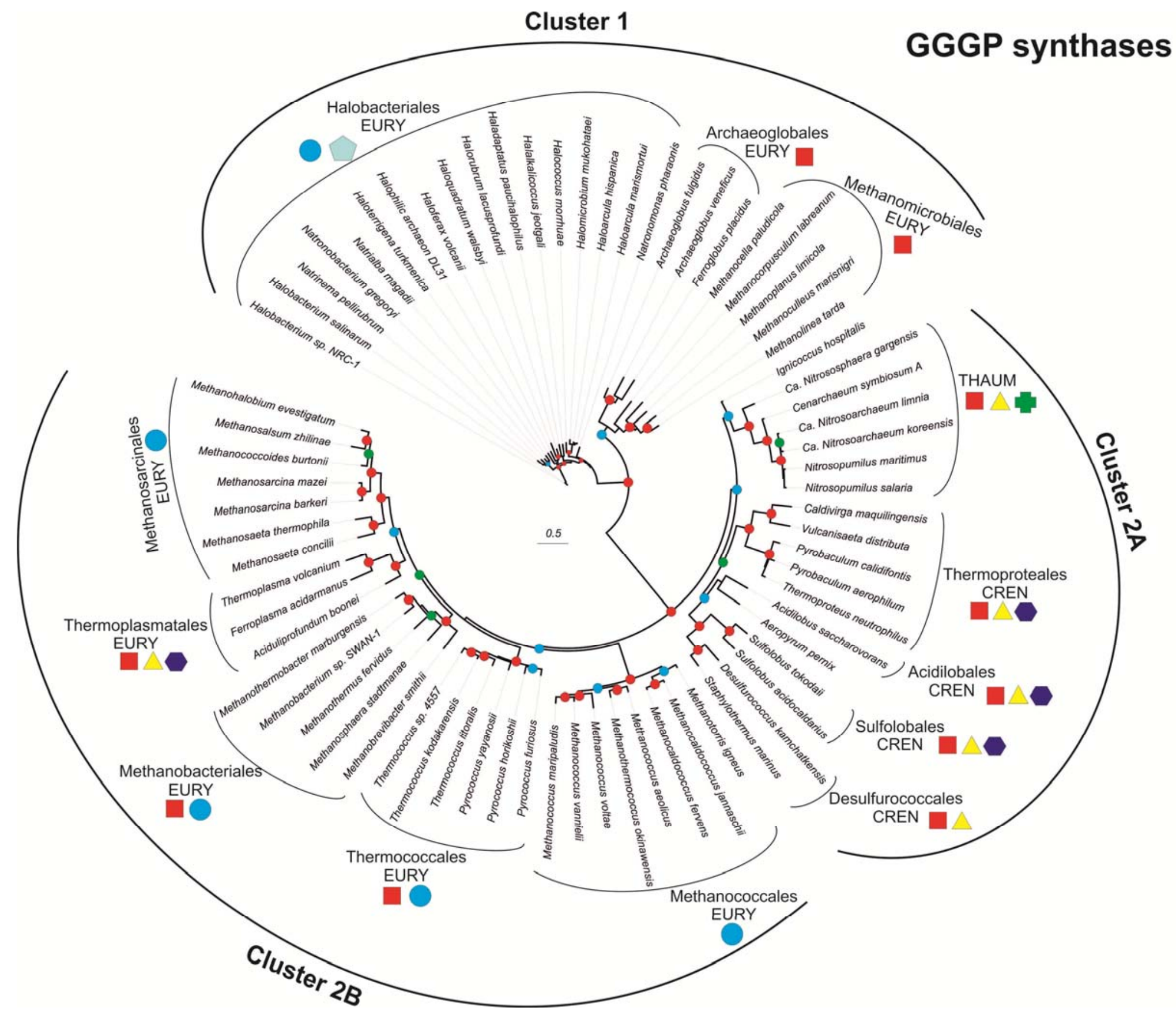




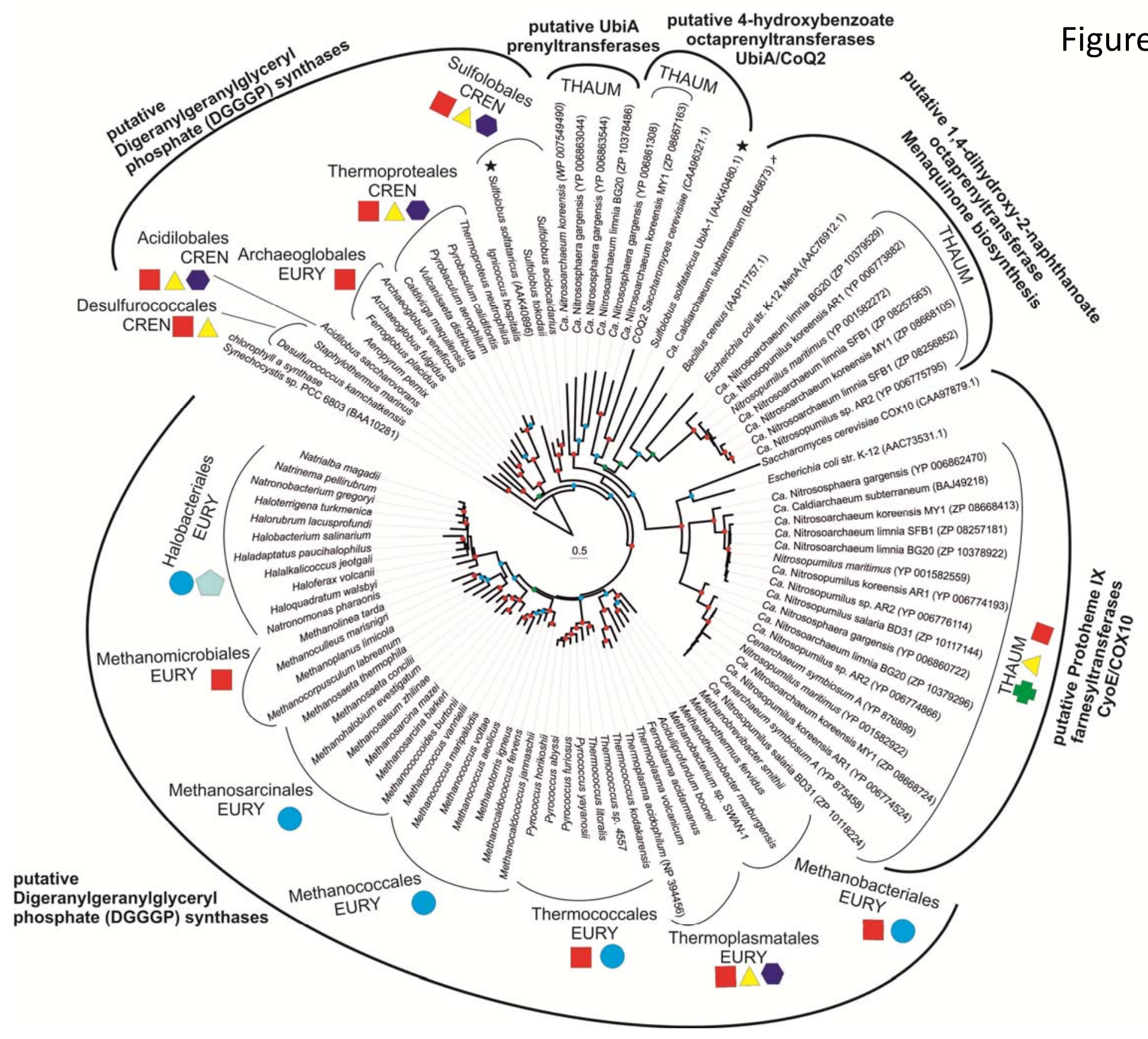




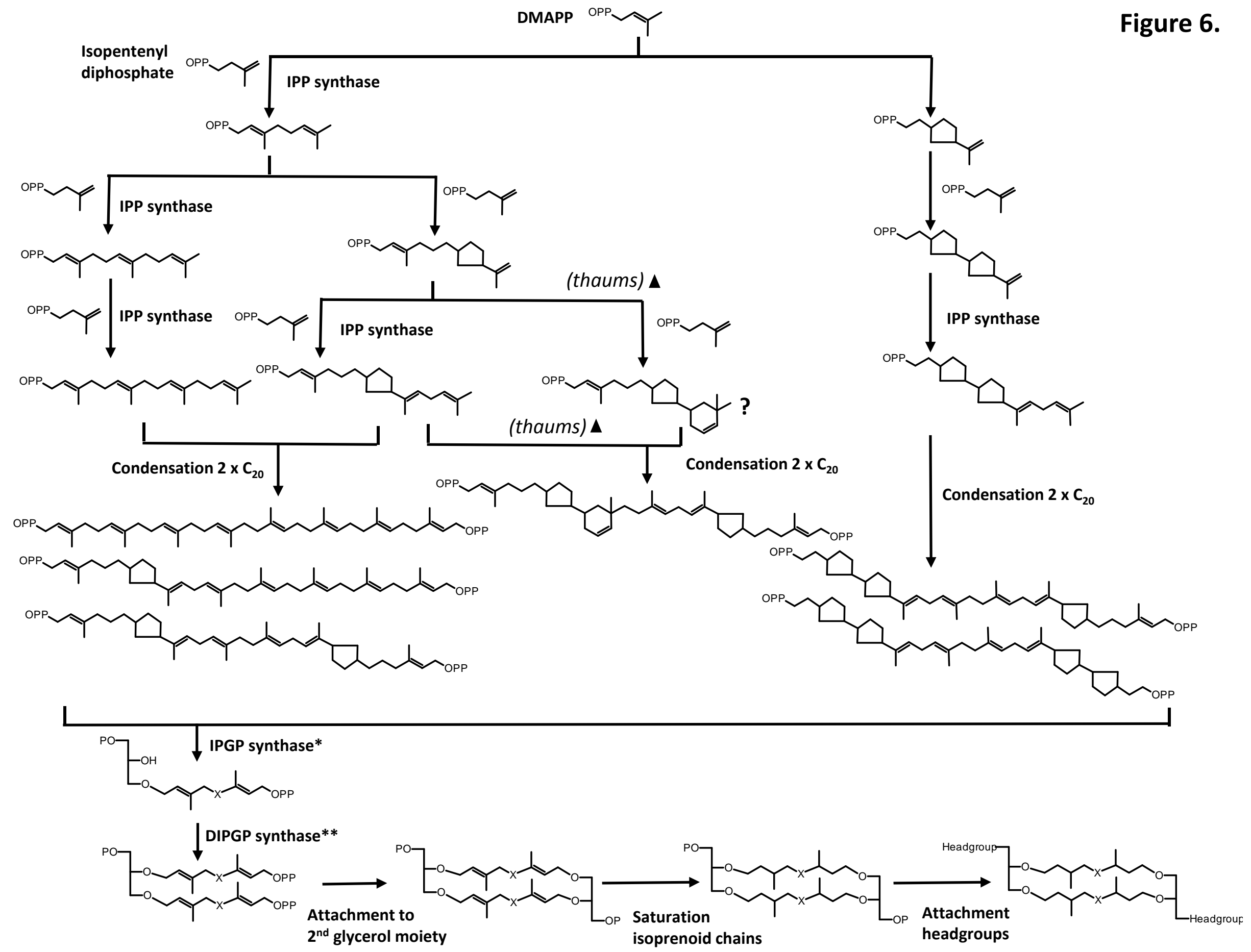

